\title{
ÍNDICE DE SUSTENTABILIDADE AGROAMBIENTAL PARA O PERÍMETRO IRRIGADO AYRES DE SOUZA
}

\author{
Environmental sustainability index for Ayres de Souza irrigated district
}

\author{
José Alves Carneiro Neto ${ }^{1}$ Eunice Maia de Andrade ${ }^{2}$,Morsyleide de Freitas Rosa ${ }^{3}$, \\ Francisco Suetônio Bastos Mota ${ }^{4}$, José Frédson Bezerra Lopes ${ }^{5}$
}

\begin{abstract}
RESUMO
Uma análise integrada do atual uso dos recursos naturais do Perímetro Irrigado de Ayres de Souza, localizado no Vale do Acaraú, Ceará, foi realizada através do desenvolvimento de um índice de sustentabilidade agroecológica. Os dados dessa pesquisa foram oriundos de questionários aplicados a 33 produtores agrícolas beneficiados pelo projeto de irrigação. Os indicadores de sustentabilidade foram estimados pelo emprego de análise fatorial, método da análise fatorial/análise de componentes principais. $\mathrm{O}$ índice de sustentabilidade estimado a partir dos indicadores selecionados registrou uma situação de sustentabilidade fragilizada ou de insustentabilidade reversível. As unidades produtivas apresentaram um porcentual de 60,6\% com alguma sustentabilidade; e os demais 39,4\% estão em condições de insustentabilidade. Os resultados também mostraram que os fatores dominantes do índice de sustentabilidade foram: nível da atividade agrícola praticada, agricultura familiar, condições atuais do sistema água-solo e infraestrutura, fontes alternativas de renda e experiência em tratos culturais.
\end{abstract}

Termos para indexação: Análise fatorial, agricultura sustentável, impacto ambiental.

\section{ABSTRACT}

The aim of this work was to make an analysis of natural resources at Ayres de Souza Irrigated District sited in Acaraú basin, Ceará, Brazil. This study was performed through an agro ecological sustainability index (ASI). The approach was based on 33 crossover form applied to small farms located in the irrigated district. Factors Analysis/Principal Components Analysis were applied to select the sustainability indicators of the irrigated district as well as to define the agro ecological sustainability index. The results showed that the Index is capable of demonstrating clear differences among productivity unit management system with respect to sustainability. $60 \%$ of the pooled productivity units presented weak sustainability and the other $39.4 \%$ were unsustainable. The dominants factors of the Index were: level of agricultural management practices, family agriculture, framework of irrigation and alternative income source

Index terms: Factorial analysis, sustainable agriculture, environmental impacts.

(Recebido em 14 de junho de 2007 e aprovado em 14 de março de 2008)

\section{INTRODUÇÃO}

Os problemas ambientais no meio rural estão aumentando à medida que novas técnicas de agricultura, apoiadas no emprego de adubos químicos, agrotóxicos e mecanização, vêm sendo empregadas desordenadamente. Além disso, o desmatamento da vegetação nativa para a utilização do solo acarreta a extinção da fauna local, e a alteração do regime das chuvas e no curso dos rios. Tal condição se torna mais agravante nas regiões secas, onde o equilíbrio dos ecossistemas é extremamente frágil.
O Semi-árido nordestino, em especial o Estado do Ceará, destaca-se por apresentar muitos problemas no que diz respeito aos recursos hídricos (MONTEIRO \& PINHEIRO, 2004). Essas regiões apresentam uma alta variabilidade temporal e espacial das precipitações, sendo que a prática da irrigação quando adequadamente empregada, pode ser uma das melhores formas de promover a ocupação e o aproveitamento econômico dos seus recursos naturais. Desde 1950, com a construção de grandes açudes (capacidade de armazenamento superior a 500 milhões de $\mathrm{m}^{3}$ ), o Departamento Nacional de Obras

\footnotetext{
'Engenheiro Civil, Mestre em Irrigação e Drenagem - Companhia de Gestão dos Recursos Hídricos/COGERH - Rua Adualdo Batista, 1550 - Cambeba 60830-080 - Fortaleza, CE - alvesneto@superig.com.br

²Engenheira Agrônoma, PhD, Professora - Departamento de Engenharia Agrícola/DENA - Universidade Federal do Ceará/UFC - Avenida Mister Hull, s/n Campus do Pici - Cx. P. 12168 - 60455-970 - Fortaleza, CE - eandrade@ufc.br

${ }^{3}$ Engenheira Química, Doutora, Pesquisadora - Embrapa Agroindústria Tropical - Rua Sara Mesquita, 2270 - Planalto Pici - 60511 -110 - Fortaleza, CE morsy@cnpat.embrapa.br

${ }^{4}$ Engenheiro Civil, Doutor, Professor - Departamento de Engenharia Agrícola/DENA - Universidade Federal do Ceará/UFC - Avenida Mister Hull, s/n Campus do Pici - Cx. P. 12168 - 60455-970 - suetonio@ufc.br

${ }^{5}$ Graduando em Agronomia - Departamento de Engenharia Agrícola/DENA - Universidade Federal do Ceará/UFC - Avenida Mister Hull, s/n - Campus do Pici - Cx. P. 12168 - 60455-970 - fredsonufc@yahoo.com.br - Bolsista do PET
} 
Contra a Seca (DNOCS) vem empregando a técnica da irrigação, em políticas sociais no meio rural das regiões semi-áridas do Nordeste brasileiro. Os distritos de irrigação contribuíram com a reforma agrária pela distribuição de lotes, porém não obtiveram satisfatório suporte do governo para a fase de operação, resultando na decadência de vários projetos. Uma das causas do insucesso de muitos projetos públicos de irrigação foi a falta de um manejo adequado, geralmente, por desconhecimento ou por falta de assistência técnica ou por ambos os fatores (MELO, 1999).

A sustentabilidade da irrigação, como parte integrante das explorações dos recursos naturais, passa por um embasamento teórico e reflexivo e acima de tudo de conscientização e treinamento de todos os que trabalham na agricultura (BRANCO, 2003). Nos dias atuais, um dos maiores desafios enfrentados pela discussão sobre o desenvolvimento sustentável é a elaboração de metodologias aplicadas que permitam avaliar a sustentabilidade de diferentes projetos, tecnologias ou agroecossistemas em situações concretas (MANGABEIRA et al., 2002; MARZALL \& ALMEIDA, 1998).

A sustentabilidade dos projetos de irrigação depende do uso da terra, geologia, qualidade da água de irrigação, drenagem natural do solo, condições climáticas locais, comercialização, nível educacional do irrigante, entre outros (MELO, 1999; WICHELNS et al., 2002). A complexidade desse sistema torna difícil estabelecer uma única variável como um indicador padrão para qualquer projeto de irrigação (GALLOPIN, 1997; TOLEDO \& NICOLELLA, 2002). Nessa busca, índices de sustentabilidade têm sido desenvolvidos para refletir resumidamente as alterações sofridas pelo meio ambiente em decorrência da agricultura irrigada. Um índice ou indicador é uma ferramenta que permite a obtenção de informações sobre uma dada realidade. Tem como principal característica a de poder sintetizar um conjunto complexo de informações, retendo apenas o significado essencial dos aspectos analisados (FOSSATTI \& FREITAS, 2004; PALÁCIO, 2004). O perímetro Ayres de Souza retrata em sua história todo o contexto dessa realidade, observandose o abandono das infra-estruturas existentes e a adoção, hoje, de um manejo ultrapassado com grandes desperdícios de água. Esse estudo se propõe ao desenvolvimento de um índice de sustentabilidade agroecológica onde se possa traçar o perfil dos irrigantes, pela realização de uma análise integrada do atual uso dos recursos naturais do perímetro irrigado de Ayres de Souza (PIAS).

\section{MATERIAL E MÉTODOS}

O Perímetro Irrigado Ayres de Souza (PIAS) está localizado no município de Sobral, Ceará (Figura 1). As suas coordenadas geográficas são: $3^{\circ} 45^{\prime}$ S e $40^{\circ} 27^{\prime} \mathrm{W}$. A implantação do perímetro irrigado foi iniciada no ano de 1974 e a sua conclusão ocorreu em 1978. Possui uma área de 615 ha irrigada por superfície, associada a 7.980 ha de áreas de sequeiro.

A coleta de dados foi realizada em julho de 2004, quando se iniciam as atividades de irrigação nos perímetros. O questionário aplicado aos irrigantes segue o padrão cross-section, com perguntas objetivas e fechadas obtendo respostas diretas, de forma a padronizar os dados.

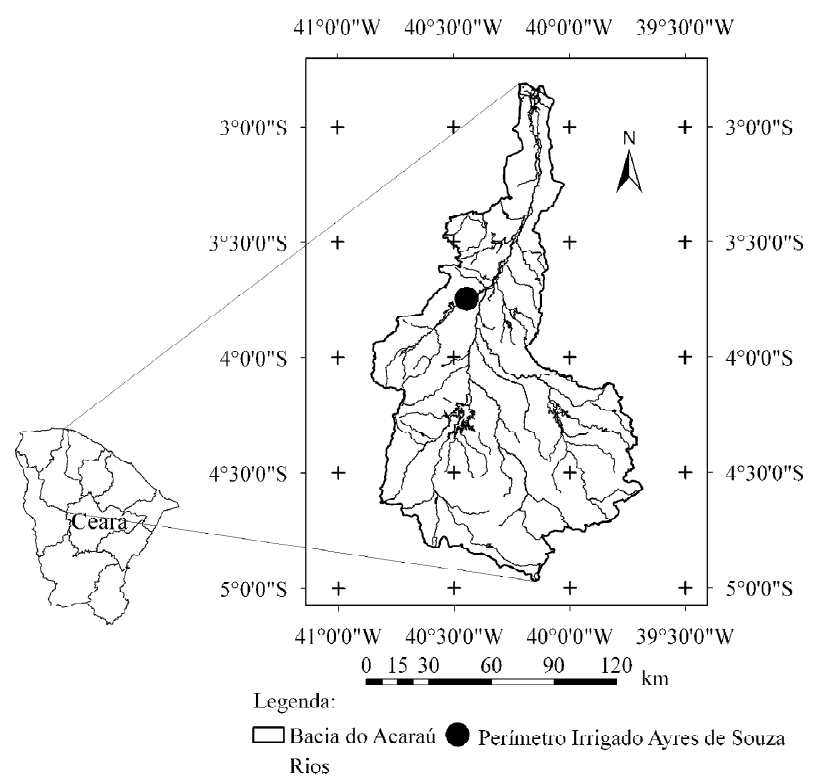

Figura 1 - Localização da área de estudo.

Considerando-se as informações da sede regional do DNOCS sobre os produtores agrícolas, optou-se por definir a quantidade de questionários aplicados a partir de uma confiança de $95 \%$, por meio da seguinte equação:

$n=\frac{N \cdot n_{0}}{N+n_{0}}$ onde, $n_{0}=\frac{z^{2} \cdot \pi \cdot(1-\pi)}{E_{0}^{2}}$

em que: $N$ (tamanho da população): 45 irrigantes; $z$ (intervalo de confiança): 0,$95 ; \pi$ (proporção na amostra): 0,$5 ; E_{\mathrm{o}}$ (erro amostral tolerável): 0,05. A população amostral proposta seria composta por 30 unidades produtivas no PIAS, porém foram aplicados 33 questionários em campo, o que reduziu o erro amostral $\left(E_{o}\right)$ para $4,25 \%$. Foi realizada uma amostragem exploratória, adotando um modelo não 
probabilístico. Por isso, as inferências extraídas da amostra devem ser ressalvadas em função das limitações desse tipo de abordagem (VERGARA, 1998).

\section{Definição do índice}

Aplicou-se o método da análise fatorial/análise de componentes principais (AF/ACP), para a construção do Índice de Sustentabilidade (IS). Essa metodologia é composta pelas seguintes etapas: preparação da matriz de correlação; extração dos fatores comuns com possível redução dos parâmetros que definem a sustentabilidade ambiental; rotação dos eixos relativos aos fatores comuns (SANDS \& PODMORE, 2000). Antes de aplicar o método para a extração de fatores, verificou-se, a partir da matriz de correlação, a adequabilidade do conjunto de variáveis ao procedimento estatístico. Nesse estudo, a adequabilidade dos dados foi aferida pelo método de Kayser Mayer Olkim, calculando-se o índice conhecido como KMO (NORUSIS, 1990). A correlação de cada variável com os fatores é expressa, em termos algébricos, por:

$X_{i}=a_{i 1} F_{1}+a_{i 2} F_{2}+\ldots+a_{i n} F_{n}+e$

em que: $X_{1}, X_{2}, \ldots, X_{i}$ (variáveis observadas); $F_{1}, F_{2}, \ldots, F_{n}$ (fatores); $a_{i 1}, a_{i 2}, \ldots, a_{i n}$ (cargas fatoriais); $e$ (erro residual). Em seguida, elaborou-se a transformação ortogonal, ou simplesmente, rotação, da matriz das cargas fatoriais. Finalmente, a determinação da matriz dos escores fatoriais, com a qual foi possível construir-se o índice para hierarquizar as observações, através da soma, simples ou ponderada, dos escores pertencentes a uma mesma observação. Os escores fatoriais de cada fator possuem distribuição normal, com média zero e variância unitária e, desse modo, podem ser utilizados para indicar a importância de cada observação no conceito expresso pelo fator (PALÁCIO, 2004).

Como os fatores captam em níveis diferentes as variâncias das variáveis, uns mais e outros menos, é razoável que, na construção de um índice envolvendo todos os fatores, seja utilizado um critério de ponderação capaz de conferir a cada fator sua importância relativa. No caso, a raiz característica, por expressar a capacidade de cada fator em captar a variância das variáveis, foi empregada como o termo de ponderação. Todas as análises estatísticas foram formuladas pelo programa SPSS, v.10 (Statistical Package for the Social Sciences), por apresentar uma boa versatilidade na manipulação dos dados, inclusive o tratamento prévio de padronização e escalonamento dos dados (NORUSIS, 1990).

Os indicadores de sustentabilidade foram obtidos da diferença dos pesos $\left(p_{i}\right)$ atribuídos a cada variável, com base nos fatores extraídos pela AF/ACP O valor do peso $\left(p_{i}\right)$ de cada variável foi ponderado em função da raiz característica de cada componente associado à explicabilidade de cada variável, em relação às componentes principais extraídas. $\mathrm{O}$ peso associado aos parâmetros do índice de sustentabilidade de cada variável foi estimado por:

$$
p_{i}=\frac{\left(F_{1} \cdot P_{i}\right)+\left(F_{2} \cdot P_{i}\right)}{\left(F_{1} \cdot \sum_{1}^{n} P_{i}\right)+\left(F_{2} \cdot \sum_{1}^{n} P_{i}\right)}
$$

em que: $p_{i}$ : peso a ser associado aos parâmetros de sustentabilidade; F: autovalor das componentes principais; $P_{i}$ : explicabilidade de cada variável em relação a componente principal.

O índice proposto retrata não só as condições de sustentabilidade local, mas também as atividades humanas que têm impactos sobre essa sustentabilidade e as medidas mitigadoras adotadas para a correção de "situações de insustentabilidade". Algebricamente, a construção do índice de sustentabilidade (IS) é a combinação, digamos linear, dos indicadores:

$\mathrm{IS}=\mathrm{p}_{1} \mathrm{I}_{1}+\mathrm{p}_{2} \mathrm{I}_{2} \ldots+\mathrm{p}_{\mathrm{i}} \mathrm{I}_{\mathrm{i}}$

em que: $I_{n}$ indicadores de sustentabilidade; P: termos de ponderação dos indicadores.

As classes dos níveis do índice desenvolvido nesse estudo fundamentaram-se nos trabalhos de Melo (1999) e Vasconcelos \& Torres Filho (1994). Como conseqüência, as unidades produtivas foram classificadas da seguinte forma:

Sustentável: ISP $<0,20$.

Sustentabilidade Ameaçada: 0,20 £ ISP < 0,40.

Sustentabilidade Comprometida: 0,40 £ ISP <0,60.

Insustentável: $0,60 £$ ISP $<0,80$.

Seriamente Insustentável: $\operatorname{ISP}^{3} 0,80$.

\section{RESULTADOS E DISCUSSÃO}

As variáveis originais, com freqüência de resposta positiva superiores a $90 \%$, foram descartadas por representarem pouco ou nenhuma diferença em termos estatísticos (MANGABEIRA et al., 2002). Os procedimentos preliminares de adequação do conjunto de variáveis à análise fatorial resultaram na eliminação de algumas variáveis, permanecendo 16 variáveis significativas (Tabela 1).

O emprego da análise fatorial pelo método dos componentes principais a essas 16 variáveis resultou em seis fatores (Tabela 1) com raízes características superiores à unidade e que, em conjunto, explicaram 70,64\% da 
Tabela 1 - Matriz de cargas fatoriais - Perímetro Irrigado Ayres de Souza, Sobral - CE, 2004.

\begin{tabular}{|c|c|c|c|c|c|c|c|}
\hline \multirow[t]{2}{*}{ Variáveis } & \multicolumn{7}{|c|}{ Componentes } \\
\hline & 1 & 2 & 3 & 4 & 5 & 6 & $C^{*}$ \\
\hline Porcentual da área de plantio & $-0,775$ & $-0,121$ & 0,087 & 0,312 & $-0,280$ & $-0,123$ & 0,814 \\
\hline Desejam receber treinamentos & 0,743 & 0,161 & 0,315 & 0,163 & $-0,078$ & $-0,090$ & 0,717 \\
\hline Os filhos possuem vinculo empregatício fora da propriedade & 0,670 & $-0,429$ & $-0,272$ & 0,094 & 0,084 & 0,025 & 0,723 \\
\hline Fertilidade do solo & 0,578 & $-0,088$ & $-0,195$ & $-0,330$ & $-0,219$ & $-0,277$ & 0,792 \\
\hline Os filhos participam das atividades agrícolas & $-0,034$ & 0,858 & $-0,241$ & $-0,149$ & $-0,161$ & 0,076 & 0,848 \\
\hline Não continuar com as atividades agrícolas & 0,175 & 0,618 & 0,256 & $-0,238$ & $-0,298$ & $-0,415$ & 0,796 \\
\hline Manejo correto dos agroquímicos & 0,153 & $-0,512$ & 0,384 & $-0,379$ & $-0,333$ & 0,325 & 0,793 \\
\hline O solo apresenta-se compactado & $-0,101$ & 0,041 & $-0,765$ & $-0,367$ & 0,098 & $-0,225$ & 0,792 \\
\hline Disponibilidade hídrica no perímetro irrigado & $-0,138$ & $-0,072$ & 0,749 & $-0,091$ & 0,209 & $-0,059$ & 0,641 \\
\hline Extrativismo de essências vegetais & $-0,110$ & $-0,177$ & 0,005 & 0,801 & 0,021 & $-0,200$ & 0,725 \\
\hline Emprego do consórcio de culturas & $-0,050$ & $-0,127$ & 0,045 & 0,577 & $-0,132$ & 0,408 & 0,538 \\
\hline Prestação de serviços temporários externo à propriedade & 0,069 & 0,363 & 0,249 & 0,494 & 0,288 & 0,111 & 0,537 \\
\hline Tempo de propriedade & 0,201 & $-0,144$ & $-0,073$ & $-0,023$ & 0,846 & $-0,061$ & 0,786 \\
\hline Preparo do solo & $-0,175$ & $-0,065$ & 0,417 & 0,055 & 0,683 & $-0,007$ & 0,679 \\
\hline Manejo dos recursos naturais & $-0,199$ & $-0,262$ & $-0,026$ & $-0,146$ & 0,135 & 0,742 & 0,699 \\
\hline Possui veículo próprio & 0,098 & 0,170 & 0,116 & 0,096 & $-0,162$ & 0,715 & 0,599 \\
\hline Raiz característica (Eigenvalues) & 2,160 & 1,921 & 1,893 & 1,860 & 1,746 & 1,723 & \\
\hline Variância (\%) & 13,499 & 12,005 & 11,830 & 11,628 & 10,913 & 10,766 & \\
\hline Variância cumulativa (\%) & 13,499 & 25,504 & 37,334 & 48,961 & 59,874 & 70,640 & \\
\hline
\end{tabular}

Fonte: elaboração própria; *C: Comunalidade

variância total das variáveis selecionadas. Pelo teste Kayser Mayer Olkim (KMO =0,563), o modelo se ajusta aos dados, uma vez que apresentou valores superiores a 0,5 . Os pesos, atribuídos às variáveis em cada fator, após a rotação ortogonal pelo critério varimax, podem ser observados na Tabela 1. A rotação dos dados foi gerada para se obter fatores com a maior ortogonalidade possível, uma vez que o objetivo principal foi medir as componentes que apresentam maior influência no sistema (HAWKINS, 1974). Procedimento semelhante foi empregado por Monteiro \& Pinheiro (2004) na seleção de critério para a implantação de tecnologias de suprimentos de água potável, em municípios cearenses afetados por alto teor de sal.

Pode-se observar que a comunalidade de cada variável foi superior a 0,5 ; ou seja, o fator correspondente reproduziu mais da metade da variância da referida variável
(NORUSIS, 1990). O total da variância, explicado por cada um dos seis fatores, apresentou porcentagem de explicabilidade semelhante, variando de 13,5 a $10,8 \%$. Isso demonstra que a sustentabilidade agroambiental apresenta dependência aproximadamente igual aos seis fatores selecionados pelo modelo da AF/ACP. O conceito de cada fator, ou seja, que aspecto o fator melhor traduz, foi definido a partir das variáveis que apresentaram maior carga fatorial (Tabela 1). Mediante a interpretação do processamento da análise fatorial, foi possível qualificar os fatores que caracterizam o meio agroambiental e as variáveis de maior contribuição (Tabela 2).

A ordem dos fatores gerados revela seu grau de importância, bem como as características inerentes aos mesmos, na perspectiva dos entrevistados. Pode-se observar que as variáveis de maior comunalidade estão relacionadas a fatores como "Produção agrícola" e 
Tabela 2 - Denominação do fator associado às variáveis explicadas - Perímetro Irrigado Ayres de Souza, Sobral - CE, 2004.

\begin{tabular}{|c|c|c|}
\hline Ordem de fatores & Denominação do fator & Variáveis ou aspectos \\
\hline \multirow{4}{*}{1} & \multirow{4}{*}{ Produção agrícola } & Porcentual da área de plantio \\
\hline & & Desejam receber treinamentos \\
\hline & & $\begin{array}{l}\text { Os filhos possuem vínculo empregatício fora da } \\
\text { propriedade }\end{array}$ \\
\hline & & Fertilidade do solo \\
\hline \multirow{3}{*}{2} & \multirow{3}{*}{ Agricultura familiar } & Os filhos participam das atividades agrícolas \\
\hline & & Não continuar com as atividades agrícolas \\
\hline & & Manejo correto dos agroquímicos \\
\hline \multirow[t]{2}{*}{3} & $\begin{array}{l}\text { Condições atuais do sistema água- } \\
\text { solo e }\end{array}$ & O solo apresenta-se compactado \\
\hline & infra-estrutura & Disponibilidade hídrica no perímetro irrigado \\
\hline \multirow{3}{*}{4} & \multirow{3}{*}{ Fontes alternativas de renda } & Extrativismo de essências vegetais \\
\hline & & Emprego do consórcio de culturas \\
\hline & & $\begin{array}{l}\text { Prestação de serviços temporários externos à } \\
\text { propriedade }\end{array}$ \\
\hline \multirow[t]{2}{*}{5} & \multirow[t]{2}{*}{ Experiência em tratos culturais } & Tempo de propriedade \\
\hline & & Preparo do solo \\
\hline \multirow[t]{2}{*}{6} & $\begin{array}{c}\text { Condições financeiras e aplicação } \\
\text { de }\end{array}$ & Manejo dos recursos naturais \\
\hline & técnicas de conservação & Possui veículo próprio \\
\hline
\end{tabular}

Fonte: elaboração própria

“Agricultura familiar". Esses resultados confirmaram os encontrados por Fossatti \& Freitas (2004) em estudos relacionados às variáveis determinantes das características sociais e econômicas da agricultura familiar no município de Santa Cruz/RS, onde foram identificados como fatores determinantes entre outros: o tamanho da família, o número de crianças, a experiência, a escolaridade e o tamanho da propriedade.

Pela Tabela 1 pode-se observar que a variável "Porcentual da área de plantio" apresentou maior peso ($0,775)$, com relação ao fator 1 "produção agrícola". Nessa variável estão inseridos aspectos técnicos e sócioeconômicos, como: adoção de novas espécies vegetais pelos irrigantes; comercialização da produção; disponibilidade e especialização da mão-de-obra; sistema de irrigação adotado; aspecto cultural da região (vocação da região para determinadas culturas e mercado regional). Mangabeira et al. (2002) mostraram que o nível de desenvolvimento de sistemas de produção apresenta uma relação direta com o nível de desenvolvimento em que se encontravam os produtores rurais.
Para o fator "Agricultura Familiar", foi observado um baixo envolvimento e uma total falta de interesse em continuar com as atividades da agricultura irrigada por parte dos filhos. Isto se explica pela dificuldade de comercialização do produto agrícola e a oferta em empregos na indústria no município de Sobral, Ceará. Foi identificada, também, a falta do uso de equipamentos de proteção individual e adequada conservação de agroquímicos (principalmente defensivos agrícolas). Os trabalhadores rurais estão expostos a riscos muito diversificados, sobretudo a exposição direta a agrotóxicos, sujeitos a graves problemas de saúde (MARZALL \& ALMEIDA, 1998).

Os fatores 4 e 6 estão intimamente relacionados à aquisição de bens provenientes da mão-de-obra agrícola, no caso, associados às condições de bem-estar social da família, como ter um veículo próprio que permita uma maior liberdade do produtor quanto ao escoamento de sua produção. Um aspecto preocupante, relacionado ao fator 4, "Fontes alternativas de renda, é a realização da prestação de serviços temporários externos à propriedade, tanto pelos 
irrigantes como por seus filhos, mostrando uma realidade financeira desassociada da atividade agrícola. Entretanto, constatou-se in-loco a existência de sistemas de cooperativas onde o irrigante, na impossibilidade de pagar por uma mão-de-obra externa, trabalha em conjunto com outros irrigantes no cultivo de suas áreas, assim, conseguem cultivar todos os lotes.

A definição do peso $\left(p_{i}\right)$, associado a cada indicador de sustentabilidade, foi obtido com base nos escores extraídos na análise fatorial/análise da componente principal (Tabela 3). $\mathrm{O}$ valor $p_{i}$ de cada indicador foi ponderado em função da raiz característica de cada fator (Tabela 1) associado à explicabilidade de cada variável em relação aos fatores extraídos (Equação 3). A soma dos valores resultantes foi igualada a um, e em seguida ponderado o valor do $p_{i}$ de cada variável, de forma que a soma de todos os $\left(p_{i}\right)$ seja igual a um (Tabela 3). Procedimento semelhante foi adotado por Palácio (2004), ao desenvolver um índice de qualidade de água para avaliar as águas do rio Trussu, Ceará.

Os maiores coeficientes do índice de sustentabilidade estão associados às variáveis "Realiza trabalhos temporários fora da propriedade" e "Desejam receber treinamento", o que indica a agricultura como única ocupação laboral. A análise de campo identificou que $88 \%$ dos irrigantes não realizam outra atividade, com dedicação exclusiva à propriedade. Por outro lado, observou-se uma certa acomodação da população quanto à adoção de novas tecnologias, confirmando o aspecto cultural, apreensivo, que dificulta o aperfeiçoamento através de cursos e treinamentos (BRANCO, 2003).

A Tabela 4 apresenta os índices de sustentabilidade gerados pela equação 4 e a classificação de cada unidade produtiva no ranking do universo estudado. Os valores negativos associados às variáveis podem ser explicados pela complexidade das questões de sustentabilidade, onde um indicador pode ter um impacto negativo sob um aspecto e positivo sob outro (GALLOPIN, 1997). A hierarquização dos IS, para cada unidade produtora, foi feita a partir de índices absolutos. Os índices de sustentabilidade obtidos variaram no intervalo de 0,002 a 1,000. Os menores valores significam níveis de maior sustentabilidade, enquanto os mais elevados IS significam níveis de menor sustentabilidade.

A média global de sustentabilidade entre os produtores situa-se em torno de 0,52 , representando um resultado mais para insustentável do que para sustentável. Para que se possa tecer maiores considerações a esse respeito, no entanto, foi preciso que algumas medidas de padronização, para a classificação dos resultados obtidos fossem tomadas. Essas medidas devem girar em torno de

Tabela 3 - Matriz de coeficientes (escores) e pesos $\left(\mathrm{p}_{\mathrm{i}}\right.$ ) associados - Perímetro Irrigado Ayres de Souza, Sobral - CE, 2004.

\begin{tabular}{lrrrrrrr}
\hline \multicolumn{1}{c}{ Variáveis } & \multicolumn{7}{c}{ Componentes } \\
\cline { 2 - 8 } & \multicolumn{1}{c}{1} & \multicolumn{1}{c}{2} & 3 & \multicolumn{1}{c}{4} & \multicolumn{1}{c}{5} & 6 & \multicolumn{1}{c}{ Peso } \\
\hline Tempo de propriedade & 0,086 & $-0,010$ & $-0,066$ & $-0,040$ & 0,494 & $-0,007$ & 0,155 \\
Percentual da área de plantio & $-0,353$ & $-0,119$ & 0,037 & 0,127 & $-0,203$ & $-0,159$ & $-0,247$ \\
Possui veículo próprio & 0,093 & 0,175 & 0,010 & 0,038 & $-0,064$ & 0,458 & 0,246 \\
Os filhos possuem vinculo empregatício & 0,323 & $-0,215$ & $-0,160$ & 0,128 & 0,016 & 0,011 & 0,051 \\
Desejo de continuar com as atividades & 0,057 & 0,265 & 0,203 & $-0,107$ & $-0,137$ & $-0,195$ & 0,046 \\
Os filhos participam das atividades agrícolas & $-0,011$ & 0,475 & $-0,125$ & $-0,052$ & 0,001 & 0,174 & 0,161 \\
Extrativismo de essências vegetais & 0,004 & $-0,117$ & $-0,058$ & 0,461 & $-0,059$ & $-0,184$ & 0,021 \\
Emprego do consórcio de culturas & 0,044 & $-0,025$ & $-0,052$ & 0,319 & $-0,110$ & 0,207 & 0,133 \\
Preparo do solo & $-0,082$ & 0,018 & 0,201 & $-0,064$ & 0,383 & $-0,025$ & 0,140 \\
O solo apresenta-se compactado & $-0,094$ & 0,002 & $-0,381$ & $-0,148$ & 0,106 & $-0,069$ & $-0,213$ \\
Manejo dos recursos naturais & $-0,079$ & $-0,044$ & $-0,058$ & $-0,139$ & 0,098 & 0,437 & 0,057 \\
Disponibilidade hídrica no perímetro & $-0,076$ & $-0,038$ & 0,423 & $-0,146$ & 0,093 & $-0,094$ & 0,055 \\
Desejam receber treinamentos & 0,372 & 0,084 & 0,172 & 0,140 & $-0,061$ & $-0,033$ & 0,262 \\
Acondicionamento dos agroquímicos & 0,050 & $-0,278$ & 0,243 & $-0,241$ & $-0,224$ & 0,119 & $-0,113$ \\
Prestação de serviços temporários externos & 0,088 & 0,247 & 0,071 & 0,253 & 0,168 & 0,094 & 0,325 \\
Fertilidade do solo & 0,235 & $-0,096$ & $-0,046$ & $-0,101$ & $-0,124$ & $-0,141$ & $-0,077$ \\
\hline
\end{tabular}

Fonte: elaboração própria. 
se estabelecer os limites que devem ser colocados sobre os índices que serão considerados mais ou menos sustentáveis, propõe Melo (1999) na estimação de um índice de agricultura sustentável: o caso da agricultura irrigada do vale do São Francisco.

Para uma melhor visualização dos resultados, procedeu-se à uma partição das unidades em classes, de acordo com sua posição relativa (Tabela 5). Para tanto, foi feita uma análise de agrupamento (Cluster Analysis), pelo método de K-médias (NORUSIS, 1990). A partir da classificação adotada, percebeu-se que apenas 15,2\% das unidades produtivas estudadas apresentam uma situação de sustentabilidade com relativo equilíbrio. Um porcentual pouco menor dos produtores $(12,1 \%)$ ainda é considerado sustentável, mas há nessa condição uma ameaça, que pode advir de qualquer um dos fatores contabilizados no índice. Porém, 33,3\% dos colonos pesquisados registraram uma sustentabilidade que já se apresenta de alguma forma

Tabela 4-Índices de sustentabilidade padronizados para as 33 unidades produtivas - Perímetro Irrigado Ayres de Souza, Sobral - CE, 2004.

\begin{tabular}{|c|c|c|c|c|c|}
\hline Unidade Produtiva & IS* & Ranking & Unidade Produtiva & IS $*$ & Ranking \\
\hline 6 & 0,002 & 1 & 20 & 0,516 & 18 \\
\hline 26 & 0,029 & 2 & 19 & 0,526 & 19 \\
\hline 17 & 0,074 & 3 & 2 & 0,581 & 20 \\
\hline 3 & 0,116 & 4 & 30 & 0,610 & 21 \\
\hline 22 & 0,119 & 5 & 9 & 0,620 & 22 \\
\hline 29 & 0,322 & 6 & 21 & 0,636 & 23 \\
\hline 24 & 0,338 & 7 & 10 & 0,674 & 24 \\
\hline 12 & 0,361 & 8 & 25 & 0,685 & 25 \\
\hline 16 & 0,370 & 9 & 13 & 0,748 & 26 \\
\hline 1 & 0,421 & 10 & 7 & 0,811 & 27 \\
\hline 5 & 0,452 & 11 & 23 & 0,825 & 28 \\
\hline 32 & 0,456 & 12 & 15 & 0,833 & 29 \\
\hline 27 & 0,457 & 13 & 8 & 0,837 & 30 \\
\hline 4 & 0,459 & 14 & 11 & 0,879 & 31 \\
\hline 31 & 0,465 & 15 & 14 & 0,989 & 32 \\
\hline 33 & 0,469 & 16 & 18 & 1,000 & 33 \\
\hline \multirow[t]{2}{*}{28} & 0,515 & 17 & Média & 0,521 & \\
\hline & & & Desvio Padrão & 0,267 & \\
\hline
\end{tabular}

Fonte: elaboração própria; *Índice de sustentabilidade

Tabela 5 - Classificação das unidades produtivas com relação à sustentabilidade do Perímetro Irrigado Ayres de Souza, 2004.

\begin{tabular}{lccc}
\hline \multicolumn{1}{c}{ Classificação } & Número de Produtores & Proporção (\%) & Proporção Acumulada \\
\hline Sustentável & 5 & 15,2 & 15,2 \\
Sustentabilidade Ameaçada & 4 & 12,1 & 27,3 \\
Sustentabilidade Comprometida & 11 & 33,3 & 60,6 \\
Insustentável & 6 & 18,2 & 78,8 \\
Seriamente Insustentável & 7 & 21,2 & 100,0 \\
Total & 33 & 100,0 & - \\
\hline
\end{tabular}

Fonte: elaboração própria. 
comprometida e os demais 39,4\% estão em condições de insustentabilidade.

Uma informação importante a ser adicionada é o fato de que $78 \%$ dos entrevistados consideraram a situação atual do perímetro entre boa e regular, o que mostra certa acomodação da população com o estado censurável em que se encontra o perímetro.

\section{CONCLUSÕES}

O modelo proposto para a criação do índice de sustentabilidade mostrou-se adequado adaptando-se bem aos indicadores sugeridos, apresentando um comportamento esperado, quanto aos resultados obtidos.

$\mathrm{O}$ índice desenvolvido demonstrou claramente a diferença entre unidades produtivas, no que concerne a sustentabilidade do modelo de exploração adotado pelos produtores.

Os fatores dominantes do índice de sustentabilidade foram: nível da atividade agrícola praticada, agricultura familiar, condições atuais do sistema água-solo e infraestrutura, fontes alternativas de renda e experiência em tratos culturais.

O índice de sustentabilidade, estimado a partir dos indicadores selecionados pelo método de componentes principais/ análise fatorial, registrou uma situação que ainda pode ser tida como otimista $(0,52)$, visto que ainda representa uma fragilizada condição de sustentabilidade ou de insustentabilidade reversível.

\section{REFERÊNCIAS BIBLIOGRÁFICAS}

BRANCO, M. C. Avaliação do conhecimento do rótulo dos inseticidas por agricultores em uma área agrícola do Distrito Federal. Revista Horticultura Brasileira, Brasília, v. 21, n. 3, p. 570-573, 2003.

FOSSATTI, D. M.; FREITAS, C. A. O caráter familiar da atividade fumageira em Santa Cruz do Sul, RS. In: CONGRESSO BRASILEIRO DE ECONOMIA E SOCIOLOGIA RURAL, 42., 2004, Cuiabá. Anais... Cuiabá: Passo, 2004. CD-ROM.

GALLOPIN, G. C. Indicators and their use: information for decision-making. In: MOLDAN, B.; BILLHARZ, S. Sustainability indicators: report of the project on indicators of sustainable development. New York: J. Wiley and Sons, 1997. cap. 1, p. 13-27.

HAWKINS, D. M. The detection of errors in multivariate data using principal components. Journal of the American Statistical Society, Alexandria, v. 69, p. 340-344, 1974.
MANGABEIRA, J. A. de C.; ROMEIRO, A. R.; AZEVEDO, E. C. de; ZARONI, M. M. H. Tipificação de sistemas de produção rural: a abordagem da análise de correspondência múltipla em Machadinho d'Oeste-RO. Campinas: Embrapa, 2002.30 p. (Embrapa monitoramento por satélite - circular técnica, 8).

MARZALL, K.; ALMEIDA, J. Parâmetros e indicadores de sustentabilidade na agricultura: limites, potencialidades e significado no contexto do desenvolvimento rural. Extensão Rural, [S.1.], v. 5, p. 25-38, 1998.

MELO, A. S. S. A. Estimação de um índice de agricultura sustentável: o caso da área irrigada do Vale do Submédio São Francisco. 1999. 167 f. Tese (Doutorado em Economia) - Universidade Federal de Pernambuco, Recife, 1999.

MONTEIRO, V. P.; PINHEIRO, J. C. V. Critério para implantação de tecnologias de suprimentos de água potável em municípios cearenses afetados pelo alto teor de sal. Revista de Economia e Sociologia Rural, Brasília, v. 42, n. 2, p. 365-387, 2004.

NORUSIS, M. J. SPSS base system user's guide. Chicago: SPSS, $1990.520 \mathrm{p}$

PALÁCIO, H. A. Q. Índice de qualidade das águas na parte baixa da bacia hidrográfica do rio Trussu Ceará. 2004. 96 f. Dissertação (Mestrado em Irrigação e Drenagem) Universidade Federal do Ceará, Fortaleza, 2004.

SANDS, G. R.; PODMORE, T. H. A generalized environmental sustainability index for agricultural systems. Agriculture, Ecosystems and Environment, Netherlands, v. 79, n. 1, p. 29-41, 2000.

TOLEDO, L. G.; NICOLELLA, G. Índice de qualidade de água em microbacia sob uso agrícola e urbano. Scientia Agrícola, Piracicaba, v. 59, n. 1, p. 181-186, 2002.

VASCONCELOS, R. R.; TORRES FILHO, W. Impactos ambientais das atividades humanas sobre a base de recursos renováveis no semi-árido: relatório preliminar. Brasília, DF: IPEA/SEPLAN, 1994.

VERGARA, S. C. Projetos e relatórios de pesquisa em administração. São Paulo: Atlas, 1998. 90 p.

WICHELNS, D.; CONE, D.; STUHR, G. Evaluating the impact of irrigation and drainage policies on agricultural sustainability. Irrigation and Drainage Systems, Netherlands, v. 16, n. 1, p. 1-14, 2002. 